\title{
Detection and Phylogenetic Analysis of Torque Teno Virus in Salivary and Tumor Biopsy Samples from Head and Neck Carcinoma Patients
}

\author{
Andrea Hettmann ${ }^{\mathrm{a}}$ Anett Demcsák ${ }^{\mathrm{b}}$ Ádám Bach ${ }^{\mathrm{c}}$ Gábor Decsi $^{\mathrm{d}}$

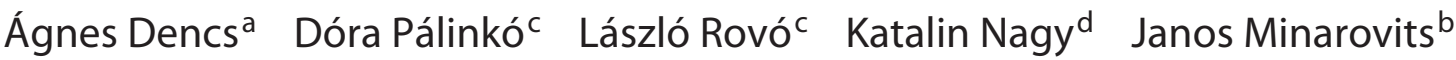 \\ Mária Takács ${ }^{\mathrm{a}}$ \\ aDivision of Virology, National Center for Epidemiology, Budapest, ${ }^{b}$ Department of Oral Biology and Experimental \\ Dental Research, Faculty of Dentistry, ' Department of Otorhinolaryngology and Head-Neck Surgery, Faculty of \\ Medicine, and d Department of Oral Surgery, Faculty of Dentistry, University of Szeged, Szeged, Hungary
}

\section{Keywords}

Torque teno virus · Head and neck cancer · Viral genotype · Phylogenetic analysis · Premalignant lesion ·

Viral carcinogenesis

\begin{abstract}
Objectives: Because torque teno virus (TTV) has been implicated in tumorigenesis as a cocarcinogen, we studied TTV prevalence in saliva and biopsy samples from head and neck cancer (HNCC) patients, patients with premalignant lesions of oral cancer, and controls. We also wished to determine the TTV genotypes in HNCC patients. Methods: A seminested polymerase chain reaction (PCR) amplifying the $\mathrm{N} 22$ region of the TTV genome, as well as direct sequencing of PCR fragments, was used. Results: TTV prevalence was higher in HNCC patients (saliva: 27/71, 38\%; tumor biopsy: 22/74, 30\%) than in controls (saliva: $8 / 56,14 \%$; oral mucosa: $1 / 19,5 \%$ ). TTV prevalence was also high in patients with premalignant lesions of oral carcinoma (saliva: 9/18, 50\%; biopsy: 5/21, 24\%). By phylogenetic analysis, TTV belonging mostly to genotypes 1 and 2 was found in HNCC patients. In most of
\end{abstract}

the cases, identical TTV strains were present in the biopsy and salivary sample of the same HNCC patient. In addition, the same TTV strain was detected in 2 laryngeal carcinoma biopsies obtained from 2 independent patients. Conclusions: Our data are compatible with the idea that TTV might act as a cocarcinogen in certain cases of HNCC. Alternatively, HNCC may facilitate either TTV replication or TTV entry into the saliva.

๑) 2016 S. Karger AG, Basel

\section{Introduction}

Torque teno virus (TTV) is a nonenveloped singlestranded DNA virus that belongs to the family Anelloviridae. TTV was discovered in the serum of a patient with non-A to $G$ hepatitis, an observation suggesting a causative role for TTV in posttransfusion hepatitis [1]. Further studies showed that TTV is highly prevalent in the human population and revealed the broad genetic diversity of the virus [2,3]. For this reason, Griffiths [4] raised the point that TTV may establish a commensal relation-

\section{KARGER}

E-Mail karger@karger.com

www.karger.com/int
(C) 2016 S. Karger AG, Basel

0300-5526/16/0592-0123\$39.50/0
Mária Takács, $\mathrm{PhD}$

National Center for Epidemiology

Albert Flórián út 2-6

HU-1097 Budapest (Hungary)

E-Mail takmar@gmail.com 
ship with the infected host. Simmonds et al. [5] also argued that TTV might be regarded as "part of the normal human flora". Although TTV causes persistent, lifelong infection and interacts with various cell types, there is no direct proof, as far as we know, of a causative role of TTV in human diseases. A higher prevalence or titer of TT viruses, however, was found to be associated with several pathological conditions such as liver and respiratory diseases, hematological disorders, and cancer, including laryngeal and hypopharyngeal carcinoma [reviewed in 3 , 6]. The exact role of TTV in these pathological conditions remains to be established.

Certain experimental data support the view that TTV may induce cell proliferation or have oncogenic properties [7]. Yokoyama et al. [8] observed impairment of renal function resembling nephrotic syndrome in transgenic mice expressing a spliced transcript from the ORF1 (open reading frame 1) gene of TTV. Renal failure was accompanied by excessive proliferation and defective differentiation of renal epithelial cells. Yokoyama et al. [8] speculated that a protein product translated from the spliced ORF1 transcript may bind to the cellular DNA and affect the pattern of gene expression [8]. A microRNA encoded by the TTV genome may also affect cell proliferation by decreasing the response to interferon signaling [9]. In addition, Rocchi et al. [10] observed that cloned DNA of a genogroup 4 TTV activated, via TLR9 (toll-like receptor 9), the expression of inflammatory cytokines in murine spleen cells [10]. An altered cytokine expression may play a role in the pathogenesis of head and neck cancer (HNCC) [11]. One may speculate that all of these TTVinduced changes may facilitate tumorigenesis.

In contrast, TAIP, a TTV-derived apoptosis-inducing protein, may block tumorigenesis or induce programmed cell death of carcinoma cells [12]. The ORF2 protein of TTV may also curb tumor development by suppressing the production of inflammatory factors activated by NF$\kappa \mathrm{B}[13]$.

Although the prevalence of TTV in the general population is high and it may be regarded as a commensal virus, certain TTV genotypes may play a role in disease development, including carcinogenesis, and it has been proposed that TTV may act as a cocarcinogen in the pathogenesis of laryngeal carcinoma [14]. For this reason, we studied the prevalence of TTV in salivary samples and tumor tissues derived from HNCC patients and compared the viral genotypes in their saliva and biopsy samples. We took advantage of the fact that a high TTV load was detected in certain bodily fluids, including saliva, that can be collected in a noninvasive way $[3,15]$.

\section{Materials and Methods}

\section{Study Design and Collection of Samples}

The study protocol was approved by the Institutional Review Board of the University of Szeged, Szeged, Hungary. Prior to the investigation, signed informed consent was obtained from each participant enrolled into this study. Saliva and tumor samples of HNCC patients and patients with premalignant lesions of oral cancer were taken from individuals attending the Department of Oto-Rhyno-Laryngology and Head-Neck Surgery, Faculty of Medicine, and the Department of Oral Surgery, Faculty of Dentistry, University of Szeged, respectively. Unstimulated whole saliva samples were collected via the simple drooling method, aliquoted, and stored at $-70^{\circ} \mathrm{C}$ until investigation. Samples from HNCC lesions and premalignant lesions including oral lichen planus and leukoplakia were taken by punch biopsy. The tissue samples were either fixed in formalin and processed for routine histopathologic examination or frozen and stored at $-70^{\circ} \mathrm{C}$ until molecular analysis. Histopathologic diagnosis of the biopsies was made at the Department of Pathology, Faculty of Medicine, University of Szeged. Control saliva and in some cases tissue (oral mucosa) samples were taken from individuals free of periodontitis, gingival inflammation, and oral mucosal disease who attended the Department of Oral Surgery, Faculty of Dentistry, University of Szeged. The sources of the samples and the characteristics of the patients involved in this study are summarized in Table 1 . The location of head and neck carcinomas is shown in Table 2. There were 29 pharyngeal and 23 laryngeal squamous cell carcinomas and 12 squamous cell carcinomas situated in the oral cavity. One lymphoepithelioma was located in the pharynx and 1 laryngeal lymphoepithelioma was also recorded. In addition, other tumors were also recorded: there were 4 metastases of unknown origin located in the lymph nodes of the head and neck region and there was 1 maxillary carcinoma and 1 piriform simus cancer. Finally, 1 maxilloethmoideal neuroendocrine carcinoma was also diagnosed (Table 2). Most of the squamous cell carcinomas studied were moderately and poorly differentiated or undifferentiated (histological grade: G2 to G4) carcinomas belonging to TNM classification stage III or IV.

\section{Sample Preparation, Polymerase Chain Reaction, and}

Sequencing

Viral DNA was extracted a using Qiagen DNA Mini Kit according to the manufacturer's instructions. Saliva samples were used directly in the kit, while tissue samples were ground in mortars before nucleic acid isolation. Samples were ground and suspended in $400 \mu \mathrm{L}$ of distilled water, and $200 \mu \mathrm{L}$ of the suspension was used directly in the kit. The presence of TTV was tested by a seminested polymerase chain reaction (PCR) performed with primers specific for the N22 region of the viral ORF1 (NG063ka CTG GCA TTT TAC CAT TTC CAA AGT T, NG059ks ACA GAC AGA GGA GAA GGC AAC ATG, and NG061bs GGC ACC ATG YTR TGG ATA GAC TGG). The PCR were carried out using REDTaq ReadyMix (Sigma) according to the manufacturer's instructions. The PCR conditions for the first PCR were $94^{\circ} \mathrm{C}$ for $3 \mathrm{~min}, 94^{\circ} \mathrm{C}$ for 30 $\mathrm{s}, 55^{\circ} \mathrm{C}$ for $30 \mathrm{~s}$, and $72^{\circ} \mathrm{C}$ for $60 \mathrm{~s}$, with a final extension at $72^{\circ} \mathrm{C}$ for $7 \mathrm{~min}$. Thirty-five amplification cycles were performed. The conditions for the second reaction were as follows: $94^{\circ} \mathrm{C}$ for $3 \mathrm{~min}$, $94^{\circ} \mathrm{C}$ for $30 \mathrm{~s}, 56^{\circ} \mathrm{C}$ for $30 \mathrm{~s}$, and $72^{\circ} \mathrm{C}$ for $60 \mathrm{~s}$, with a final extension at $72^{\circ} \mathrm{C}$ for $7 \mathrm{~min}$. Twenty-five amplification cycles were per- 
Table 1. Sample sources and patient characteristics

\begin{tabular}{|c|c|c|c|c|c|c|}
\hline & \multicolumn{2}{|c|}{$\begin{array}{l}\text { Head and neck cancer } \\
\text { patients }\end{array}$} & \multicolumn{2}{|c|}{$\begin{array}{l}\text { Patients with premalignant } \\
\text { lesions of oral cancer }\end{array}$} & \multicolumn{2}{|c|}{ Controls } \\
\hline & saliva & tumor biopsy & saliva & lesion biopsy & saliva & oral mucosa \\
\hline Samples, $n$ & $69^{\mathrm{a}}$ & 72 & $18^{\mathrm{b}}$ & 21 & 58 & $21^{\mathrm{c}}$ \\
\hline Mean age of the patients, years & 60 & 59.9 & 61 & 62.7 & 35.8 & 38.4 \\
\hline Male-to-female ratio & $6: 1$ & $6: 1$ & $1: 3$ & $1: 3$ & $1: 1$ & $1: 1$ \\
\hline
\end{tabular}

a Sixty-nine saliva and related tumor biopsy sample pairs. ${ }^{\mathrm{b}}$ Eighteen saliva and premalignant biopsy sample pairs. ${ }^{c}$ Twenty-one saliva and control oral mucosa sample pairs.

Table 2. Location of the head and neck carcinomas analyzed

\begin{tabular}{lll}
\hline & Tumors at the site, $n$ & Note \\
\hline $\begin{array}{l}\text { Tumor location } \\
\text { Pharynx }\end{array}$ & 30 & 29 HNSCC \\
Larynx & 23 & 1 lymphoepithelioma \\
& 12 & 2 HNSCC \\
Oral cavity & 1 lymphoepithelioma \\
Other & 12 HNSCC \\
& 4 lymph node metastases of unknown origin \\
& 1 maxillary carcinoma \\
& 1 pyriform sinus cancer \\
& 1 maxilloethmoideal cancer, neuroendocrine \\
\hline
\end{tabular}

HNSCC, head and neck squamous cell carcinoma.

formed. The PCR products were visualized using the GR Safe Nucleic Acid Gel Stain $(\times 10,000$ in water) and UV lighting after agarose gel electrophoresis.

In the case of saliva samples, 2-fold serial dilution of the DNA of TTV-positive samples was performed and the same PCR cycles were done once again. The most diluted sample that showed positivity was used in the sequencing reaction; thus, the most abundant TTV strain was amplified and could be sequenced directly. In the case of tumor samples, this procedure was not necessary as all of the tumor samples could be sequenced directly.

PCR products were purified using the Viogene Advanced ${ }^{\mathrm{TM}}$ PCR Clean Up System. The purified PCR products were sequenced directly from both directions using a ABI BigDye Terminator v3.1 Cycle Sequencing Kit (Applied Biosystems) according to the manufacturer's protocol. Ethanol precipitation of cycle-sequenced products was performed. Cleaned sequenced templates were resuspended in Hi-Di formamide (Applied Biosystems) and separated and analyzed on an ABI 3500 capillary sequencer. Equivocal sequences, mostly resulting from multiple TTV infections, were excluded from further analysis. Fourteen TTV samples obtained from tumors and 11 from saliva were included in this study, and among these there were 11 tumor-saliva sample pairs. The obtained sequences were deposited in GenBank with the accession numbers KU720361-KU720375 and KX371259-KX371268.

\section{Phylogenetic Analysis}

Manually checked sequences were compared to GenBank database entries using the BLAST program. Reference sequences were downloaded from GenBank (accession No. AY456101, AJ309728, AJ402218, AF060547, AB008394, AB017772, AB017774, $\mathrm{AB} 017775, \mathrm{AB} 017776, \mathrm{AB} 017777, \mathrm{AB} 037926, \mathrm{AF} 261761$, AB017779, AB054647, AB017778, and AB008394), as well as sequences obtained from previous Hungarian studies [16, 17] (accession No. FN689754, FN689755, FN689760, FN689770, FN689775, FN689782, FN689785, FN689790, FN689791, AJ510252, AJ510254, AJ510258, AJ510261, and AJ510262). The sequences obtained from patients and reference sequences from GenBank were aligned with Multalin. The phylogenetic analyses were conducted using $M E G A$ version 6 via the neighbor-joining method and a Kimura 2-parameter nucleotide substitution model with bootstrapping of 1,000 repetitions.

\section{Statistics}

Statistical analyses were carried out using SPSS v20 software with Fisher's exact test. 
Table 3. TTV prevalence in head and neck cancer patients, patients with premalignant lesions of oral cancer, and controls

\begin{tabular}{ll}
\hline Head and neck cancer patients & \\
$\quad$ Saliva & $27 / 69(39.1)$ \\
Tumor biopsy & $22 / 72(30.5)$ \\
Patients with premalignant lesions of oral cancer & \\
$\quad$ Saliva & $9 / 18(50)$ \\
$\quad$ Lesion biopsy & $5 / 21(23.8)$ \\
Controls & \\
$\quad$ Saliva & $8 / 58(13.8)$ \\
Oral mucosa & $1 / 21(4.8)$ \\
\hline
\end{tabular}

${ }^{a}$ Values are expressed as the number of TTV-positive samples/ total number of samples with percentages in parentheses.

\section{Results}

Using seminested TTV PCR, we found a significantly higher TTV prevalence in the salivary samples of HNCC patients $(27 / 69 ; 39 \%)$ than in control saliva samples $(8 / 56 ; 14 \%, p<0.005)$. Similarly, TTV prevalence was significantly higher in the tumor biopsy samples of HNCC patients $(22 / 72 ; 30.5 \%)$ compared to the available oral mucosa samples taken from control patients (1/19; $5 \%$; $p<0.03)$. The TTV prevalence in cases with oral premalignant lesions was also higher in the saliva samples $(9 / 18 ; 50 \%)$ compared to the controls $(p<0.04)$. The TTV prevalence in oral premalignant lesion biopsy samples $(5 / 21 ; 23.8 \%)$ did not differ, however, significantly from that of control oral mucosa samples $(p<0.18)$, possibly due to the relatively low number of samples. The TTV prevalence rates are summarized in Table 3. Neither the location of the tumors nor the grade or stage of the carcinomas showed a significant correlation with TTV prevalence as assessed by seminested PCR (data not shown).

Fourteen TTV strains found in different HNCC biopsies and 11 of the related saliva samples were sequenced. The biopsy samples were derived from 4 laryngeal squamous cell carcinomas (carrying TTV strains KU720363, KU720366, KU720367, and KX371265), 5 pharyngeal carcinomas (carrying TTV strains KU720361, KU720368, KU720369, KX371264, and KX371267), and 3 oropharyngeal carcinomas (carrying TTV strains KU720364, KU720365, and KX371266) (Fig. 1). In addition, there was a sample from a sinonasal squamous cell carcinoma (carrying TTV strain KU720362) and another derived from a neuroendocrine carcinoma located in the maxillary and ethmoideal sinuses (carrying TTV strain
KX371268) (Fig. 1). Two of the tumor-associated TTV sequences (KU720365 and KU720366) lacked a corresponding salivary sequence pair, and ambiguous TTV sequences were found in the salivary sample of a patient with a TTV-positive tumor (KU720368); thus, only 11 salivary TTV sequences were used for phylogenetic analysis. All of the TTV strains found in the HNCC biopsy samples and in saliva samples of HNCC patients belonged to genotypes 1,2, and 3 according to the old classification system (Fig. 1). These TTV genotypes were identified earlier in Hungary either in healthy individuals or in different patient groups including patients with malignant lesions $[16,17]$ (Fig. 1). As a matter of fact, in our study the majority of the TTV sequences were of genotype 1 or 2; only 1 saliva-tumor pair (KU720362 and KU720370) belonged to genotype 3 . In 9 cases the same TTV strain was present both in saliva and in tumor samples; in 2 cases, however, different strains were detected. We also noticed that the TTV strain detected in the biopsy of a laryngeal carcinoma (shown as KU720363 tumor56 LSCC in Fig. 1) and in the salivary sample of the same patient (KU720372 saliva56 LSCC) was identical to the TTV strain detected in the tumor biopsy sample derived from another patient with laryngeal carcinoma (shown as KU720366 tumor62 LSCC in Fig. 1). This finding is compatible with the idea that certain genogroups, genotypes, or species of the genetically diverse TTV may play a role in the development of laryngeal carcinoma.

\section{Discussion}

TTV genomes show a high degree of genetic variability and they were traditionally divided into 5 phylogenetic clusters or genetic groups which show at least $50 \%$ nucleotide sequence divergence. Furthermore, each group consists of several genotypes separated by more than $30 \%$ sequence difference from one another [reviewed in $3,18,19]$. Hijikata et al. [20] suggested that the high genetic diversity of TTV could possibly be explained by a long coevolution of TTV with humans and adaptation of the virus to its host. Although TTV infects a variety of tissues and cell types, and its cellular receptor remains to be identified, the presence of different viral genotypes in plasma and peripheral blood mononuclear cells suggests that TTV variants may preferentially replicate or accumulate in distinct body compartments [21]. In addition, coinfection of the same individual with different TTV genotypes has also been recorded at various geographical locations $[16,18,21,22]$. Because genetic 
Fig. 1. Phylogenetic analysis of TTV strains detected in saliva and tumor biopsy samples of head and neck cancer patients. The phylogenetic tree is based on the N22 segment of the TTV genome and it was constructed with Mega6 software using Kimura's 2-parameter model. Strains found in saliva are indicated by squares, whereas strains found in tumor tissues are indicated by triangles. Different sequences are indicated by the accession number, the type of sample, and a number that refers to the code of the patient. Abbreviations refer to the location of tumors. LSCC, laryngeal squamous cell carcinoma; OSCC, oral squamous cell carcinoma; PSCC, pharyngeal squamous cell carcinoma; OLP, oral lichen planus; OL, oral leukoplakia. "Other" refers to either a sinonasal squamous cell carcinoma (saliva52, tumor52) or a neuroendocrine carcinoma located in the maxillary and ethmoideal sinuses (saliva82, tumor82). Sequences obtained earlier in Hungary $[16,17]$ are indicated by diamonds. Sequences from healthy persons [16] are marked hun after T. A genotype 14 (gt14) sequence was used as an outgroup to root the tree. Numbers on the nodes indicate bootstrap values (\%). The rectangle indicates that the same TTV strain was detected in 2 laryngeal carcinoma biopsies obtained from 2 independent patients.

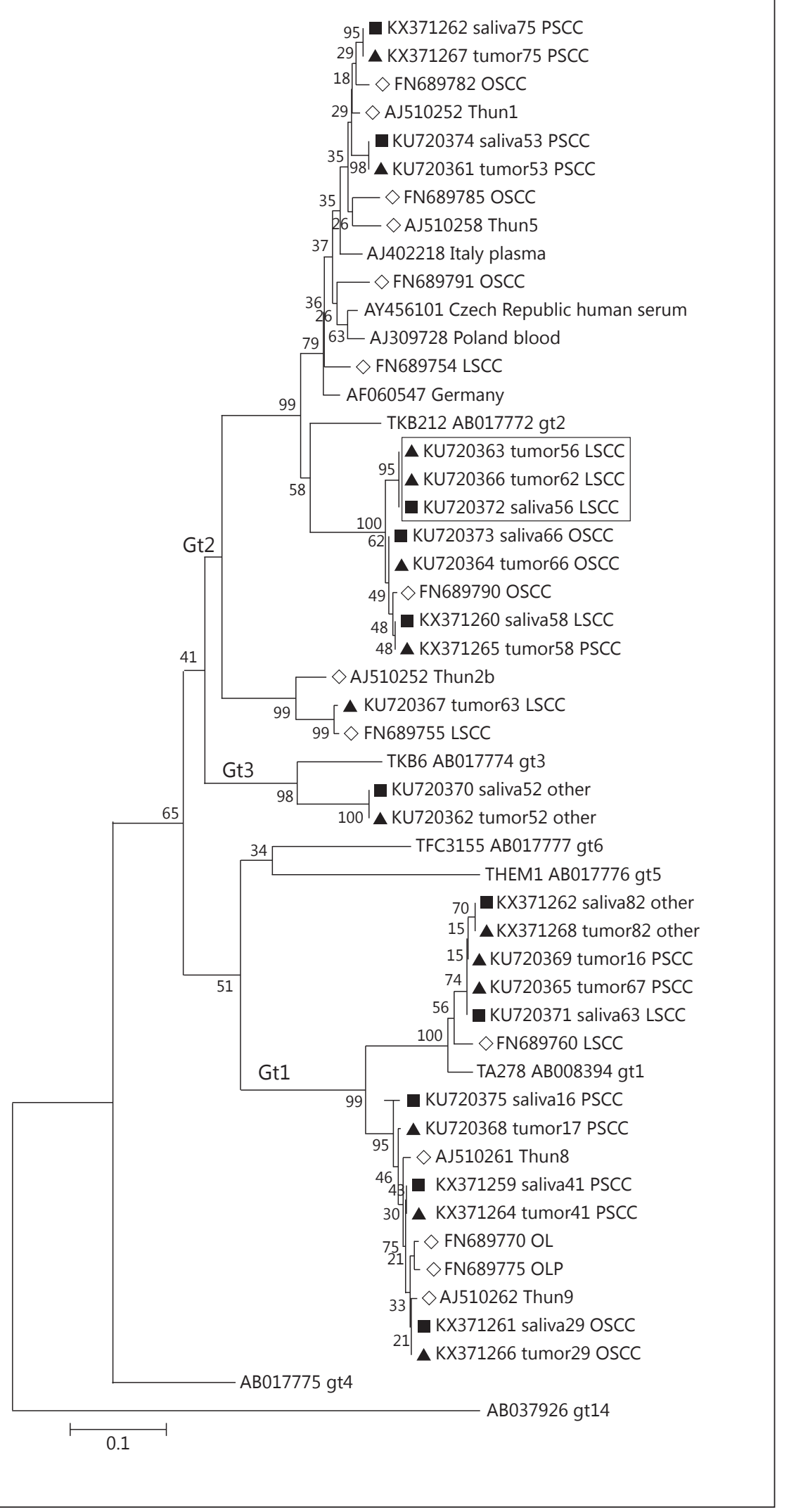


differences between DNA virus variants may result in different tissue tropisms and an altered pathogenicity [23, 24 ], one may speculate that certain genogroups, genotypes, or species of the genetically diverse TTV also play a role in disease development [3]. With respect to HNCC, it has been proposed that TTV may act as a cocarcinogen in the pathogenesis of laryngeal carcinoma [14].

We found a significantly higher TTV prevalence in saliva $(27 / 71 ; 38 \%)$ and tumor biopsy $(22 / 74 ; 30 \%)$ samples of HNCC patients than in control samples (saliva: $8 / 56$, $13.8 \%$; oral mucosa: $1 / 19,5 \%$; Table 3 ). In addition, we found a high TTV prevalence in patients with premalignant lesions predisposing to the development of oral carcinoma (saliva: $9 / 18,50 \%$; biopsy: $5 / 21,24 \%$ ). We noticed that in our study the TTV prevalence in the saliva of controls (13.8\%) was comparable with the TTV positivity rate of sera derived from healthy individuals $(20 / 108 ; 18.5 \%)$ observed in a previous study performed using the same seminested PCR assay in Hungary [16]. Although the HNCC patient group and the controls were not age matched in the present study, we do not think that age had a significant impact on TTV prevalence, because others observed no significant difference in TTV prevalence between consecutive age groups above 30 years of age [25-27]. Our data suggest that TTV may replicate in certain HNCC neoplasms and oral precancerous lesions and it is transmitted to the saliva of the patients. Alternatively, TTV replicating in various cell types of HNCC patients may have increased access to the saliva, compared to control individuals.

Our phylogenetic analysis showed that the TTV strains detected in HNCC patients belonged to genotype 1, 2, or 3 , similarly to the TTV strains identified earlier in healthy individuals and various patient groups in Hungary [14, 16, 17, 28, 29] (Fig. 1). The TTV genotypes 1, 2, and 3 belong to genogroup 1 according to the former classification used in most TTV phylogenetic studies, and all of them are included in species TTV1 of the novel classification system [17]. Comparison of the TTV sequences in saliva/tumor pairs derived from the same individual showed that in 9 out of 11 cases the TTV strain detected in the tumor biopsy was identical to the one found in the salivary sample of the same HNCC patient. In 2 cases, however, the sequences differed (samples from a pharyngeal carcinoma patient, referred to as KU7202369 tumor16 and KU720376 saliva16 in Fig. 1, and samples from a laryngeal carcinoma patient, referred to as KU720367 tumor63 and KU720371 saliva63 in Fig. 1), suggesting the occurrence of mixed infections and possible compartmentalization of the TTV strains detected.
Our data revealed that both genotype 1 and genotype 2 TTV strains could infect both laryngeal carcinomas and pharyngeal carcinomas, and they could be detected in the saliva of such patients as well. The only genotype 3 TTV strain detected was present in the tumor biopsy and salivary sample of a patient with sinonasal squamous cell carcinoma (shown as KU720362 tumor52 and KU720370 saliva52 in Fig. 1). We found, based on sequencing of the N22 region of ORF1, that the same TTV strain was present in 2 laryngeal carcinoma biopsies obtained from 2 independent patients (KU720363 tumor56 LSCC and KU720366 tumor62 LSCC; Fig. 1). This observation supports the idea that certain TTV genogroups, genotypes, or species may play a role in disease development including the development of laryngeal carcinoma $[3,14]$.

Although several studies have demonstrated the presence of TTV in saliva, a bodily fluid that can be collected in a noninvasive way $[15,19,30-33]$, the presence and genogroup/genotype distribution of TTV in salivary samples and in the corresponding tumor biopsies of HNCC patients have not been studied before. Our study supports the view that the use of saliva samples may contribute to the molecular epidemiological characterization of TTV infection and TTV-associated diseases.

The samples analyzed in our study were collected from Southeast Hungary. The distribution of genotypes found in this region of the country was similar to those found earlier in Hungary $[16,17]$ and the Czech Republic, another country in Central Europe [27].

Szládek et al. [14] noticed that coinfection with genogroup 1 TTV and HPV was associated with a poor clinical outcome of laryngeal carcinoma. A follow-up study demonstrated a high coprevalence of these viruses in lesions of oral squamous cell cancer and oral lichen planus compared to healthy mucosa [29]. Our observation that the TTV prevalence was high in patients with lesions associated with a high risk of oral cancer is compatible with the idea that TTV may play a role in oral carcinogenesis. Alternatively, TTV may preferentially target precancerosus or inflammatory lesions such as lichen planus and it may replicate in such lesions. One may speculate that, in addition to epithelial cells, T cells could also be potential sites of TTV replication [34]. Further research may elucidate the role of TTV in stepwise carcinogenesis. 


\section{References}

1 Nishizawa T, Okamoto H, Konishi K, Yoshizawa H, Miyakawa Y, Mayumi M: A novel DNA virus (TTV) associated with elevated transaminase levels in posttransfusion hepatitis of unknown etiology. Biochem Biophys Res Commun 1997;241:92-97.

2 Davidson I, Shulman LM: Unraveling the puzzle of human anellovirus infections by comparison with avian infections with the chicken anemia virus. Virus Res 2008;137:115.

3 Spandole S, Cimponeriu D, Berca LM, Mihaescu G: Human anelloviruses: an update of molecular, epidemiological and clinical aspects. Arch Virol 2015;160:893-908.

4 Griffiths P: Time to consider the concept of a commensal virus? Rev Med Virol 1999;9:7374.

5 Simmonds P, Prescott LE, Logue C, Davidson F, Thomas AE, Ludlam CA: TT virus - part of the normal human flora? J Infect Dis 1999; 180:1748-1750.

6 de Villiers EM, Schmidt R, Delius H, zur Hausen H: Heterogeneity of TT virus related sequences isolated from human tumour biopsy specimens. J Mol Med (Berl) 2002;80:44-50.

7 zur Hausen $\mathrm{H}$, de Villiers EM: TT viruses: oncogenic or tumor-suppressive properties? Curr Top Microbiol Immunol 2009;331:109116.

8 Yokoyama H, Yasuda J, Okamoto H, Iwakura Y: Pathological changes of renal epithelial cells in mice transgenic for the TT virus ORF1 gene. J Gen Virol 2002;83:141-150.

9 Kincaid RP, Burke JM, Cox JC, de Villiers EM, Sullivan CS: A human torque teno virus encodes a microRNA that inhibits interferon signaling. PLoS Pathog 2013;9:e1003818.

10 Rocchi J, Ricci V, Albani M, Lanini L, Andreoli E, Macera L, Pistello M, Ceccherini-Nelli L, Bendinelli M, Maggi F: Torque teno virus DNA drives proinflammatory cytokines production and secretion by immune cells via toll-like receptor 9. Virology 2009;394:235242.

11 Wang F, Arun P, Friedman J, Chen Z, Van Waes C: Current and potential inflammation targeted therapies in head and neck cancer. Curr Opin Pharmacol 2009;9:389-395.

12 Kooistra K, Zhang YH, Henriquez NV, Weiss B, Mumberg D, Noteborn MH: TT virusderived apoptosis-inducing protein induces apoptosis preferentially in hepatocellular carcinoma-derived cells. J Gen Virol 2004;85: 1445-1450.
13 Zheng H, Ye L, Fang X, Li B, Wang Y, Xiang X, Kong L, Wang W, Zeng Y, Ye L, Wu Z, She $\mathrm{Y}$, Zhou X: Torque teno virus (SANBAN isolate) ORF2 protein suppresses NF-kappaB pathways via interaction with IkappaB kinases. J Virol 2007;81:11917-1124.

14 Szládek G, Juhász A, Kardos G, Szoke K, Major T, Sziklai I, Tar I, Márton I, Konya J, Gergely L, Szarka K: High co-prevalence of genogroup 1 TT virus and human papillomavirus is associated with poor clinical outcome of laryngeal carcinoma. J Clin Pathol 2005;58: 402-405.

15 Deng X, Terunuma H, Handema R, Sakamoto M, Kitamura T, Ito M, Akahane Y: Higher prevalence and viral load of TT virus in saliva than in the corresponding serum: another possible transmission route and replication site of TT virus. J Med Virol 2000;62:531-537.

16 Takács M, Balog K, Tóth G, Balogh Z, Szomor KN, Brojnás J, Rusvai E, Minárovits J, Berencsi G: TT virus in Hungary: sequence heterogeneity and mixed infections FEMS Immunol Med Microbiol 2003;35:153-157.

17 Fehér E, Kardos G, Gáll T, Kis A, Gergely L, Szarka K: Comparison of diversity of Torque teno virus 1 in different mucosal tissues and disorders. Acta Microbiol Immunol Hung 2011;58:319-337.

18 Ball JK, Curran R, Berridge S, Grabowska AM, Jameson CL, Thomson BJ, Irving WL, Sharp PM: TT virus sequence heterogeneity in vivo: evidence for co-infection with multiple genetic types. J Gen Virol 1999;80:17591768.

19 Gallian P, Biagini P, Zhong S, Touinssi M, Yeo W, Cantaloube JF, Attoui H, de Micco P, Johnson PJ, de Lamballerie X: TT virus: a study of molecular epidemiology and transmission of genotypes 1, 2 and 3. J Clin Virol 2000;17:43-49.

20 Hijikata M, Takahashi K, Mishiro S: Complete circular DNA genome of a TT virus variant (isolate name SANBAN) and 44 partial ORF2 sequences implicating a great degree of diversity beyond genotypes. Virology 1999; 260:17-22.

21 Okamoto H, Kato N, Iizuka H, Tsuda F, Miyakawa Y, Mayumi M: Distinct genotypes of a nonenveloped DNA virus associated with posttransfusion non-A to $\mathrm{G}$ hepatitis (TT virus) in plasma and peripheral blood mononuclear cells. J Med Virol 1999;57:252-258.

22 Niel C, Saback FL, Lampe E: Coinfection with multiple TT virus strains belonging to different genotypes is a common event in healthy Brazilian adults. J Clin Microbiol 2000;38: 1926-1930.
23 zur Hausen H: Papillomaviruses in the causation of human cancers - a brief historical account. Virology 2009;384:260-265.

24 Lorincz AT, Reid R, Jenson AB, Greenberg MD, Lancaster W, Kurman RJ: Human papillomavirus infection of the cervix: relative risk associations of 15 common anogenital types. Obstet Gynecol 1992;79:328-337.

25 Haloschan M, Bettesch R, Görzer I, Weseslindtner L, Kundi M, Puchhammer-Stöckl E: TTV DNA plasma load and its association with age, gender, and HCMV IgG serostatus in healthy adults. Age (Dordr) 2014;36:9716.

26 Saback FL, Gomes SA, de Paula VS, da Silva RR, Lewis-Ximenez LL, Niel C: Age-specific prevalence and transmission of TT virus. J Med Virol 1999;59:318-322.

27 Saláková M, Nemecek V, König J, Tachezy R: Age-specific prevalence, transmission and phylogeny of TT virus in the Czech Republic. BMC Infect Dis 2004;4:56.

28 Dencs A, Hettmann A, Szomor KN, Kis Z, Takács M: Prevalence and genotyping of group 3 torque teno viruses detected in health care workers in Hungary. Virus Genes 2009; 39:39-45.

29 Fehér E, Gáll T, Murvai M, Kis A, Boda R, Sápy T, Tar I, Gergely L, Szarka K: Investigation of the occurrence of torque teno virus in malignant and potentially malignant disorders associated with human papillomavirus. J Med Virol 2009;81:1975-1981.

30 Ross RS, Viazov S, Runde V, Schaefer UW, Roggendorf M: Detection of TT virus DNA in specimens other than blood. J Clin Virol 1999; 13:181-184.

31 Goto K, Sugiyama K, Ando T, Mizutani F, Terabe K, Tanaka K, Nishiyama M, Wada Y: Detection rates of TT virus DNA in serum of umbilical cord blood, breast milk and saliva. Tohoku J Exp Med 2000;191:203-207.

32 Inami T, Konomi N, Arakawa Y, Abe K: High prevalence of TT virus DNA in human saliva and semen. J Clin Microbiol 2000;38:24072408.

33 Matsubara H, Michitaka K, Horiike N, Yano M, Akbar SM, Torisu M, Onji M: Existence of TT virus DNA in extracellular body fluids from normal healthy Japanese subjects. Intervirology 2000;43:16-19.

34 Focosi D, Macera L, Boggi U, Nelli LC, Maggi F: Short-term kinetics of torque teno virus viraemia after induction immunosuppression confirm $\mathrm{T}$ lymphocytes as the main replication-competent cells. J Gen Virol 2015;96: 115-117. 